\title{
Comment on: "Adverse Drug Reactions and Clinical Outcomes in Patients Initiated on Antiretroviral Therapy: A Prospective Cohort Study from Ethiopia”
}

\author{
Mark Cotton $^{1} \cdot$ Simone Nicol ${ }^{1}$
}

Published online: 5 August 2015

(C) Springer International Publishing Switzerland 2015

Dear Editor,

We read with interest the recent article published in Drug Safety by Bezabhe and colleagues [1] on the linkage between adverse drug reactions (ADRs) and poor adherence in patients initiating antiretroviral therapy (ART) in two clinics in Ethiopia.

As the ART rollout expands in Africa, attention must focus on maximizing benefits and prolonging efficacy. For both of these goals, adherence to therapy is a key factor. Detecting failure of first-line ART requires measurement of plasma human immunodeficiency virus (HIV) RNA levels and genotyping to guide subsequent therapy in many cases, with second-line options being more expensive than firstline ART. Persisting with a failing regimen increases the likelihood of resistance [2].

More than 2 million serious ADRs, with 100,000 deaths, occur each year [3]. In their prospective study, Bezabhe and colleagues [1] found that more than $85 \%$ of the cohort experienced at least one ADR, and almost a third experienced a severe ADR in the first 12 weeks of therapy. Most ADRs were already evident within 4 weeks. Patients with severe ADRs were less likely to be adherent in this study. Lack of increase in body mass index and unemployment were linked to ADRs.

Simone Nicol

simnicol@gmail.com

Mark Cotton

mcot@sun.ac.za

1 Department of Paediatrics and Child Health, Faculty of Medicine and Health Sciences, Stellenbosch University, Stellenbosch, South Africa
The message is clear. ADRs need to be actively sought at the first follow-up visit and then for the first 3 months. Interventions can be rapidly implemented. Unemployed patients and those without weight gain (an easy surrogate for body mass index) are most vulnerable. The intervention can be rapidly employed through heightened sensitivity of healthcare workers in busy clinics and with well-trained counsellors strategically placed in the clinic.

\section{Compliance with Ethical Standards}

Funding No sources of funding were used in the preparation of this letter.

Conflict of interest Mark Cotton and Simone Nicol have no conflicts of interest that are directly relevant to the content of this letter.

\section{References}

1. Bezabhe WM, Bereznicki LR, Chalmers L, Gee P, Kassie DM, Bimirew MA, Peterson GM. Adverse drug reactions and clinical outcomes in patients initiated on antiretroviral therapy: a prospective cohort study from Ethiopia. Drug Saf. 2015;38(7):629-39. doi:10.1007/s40264-015-0295-7.

2. World Health Organization (WHO). Consolidated guidelines on the use of antiretroviral drugs for treating and preventing HIV infection: recommendations for a public health approach. Geneva: WHO; 2013. http://www.who.int/hiv/pub/guidelines/arv2013/en/. Accessed 20 July 2015.

3. US Food and Drug Administration (FDA). Preventable adverse drug reactions: a focus on drug interactions. Silver Spring: FDA; 2014. http://www.fda.gov/Drugs/DevelopmentApprovalProcess/ DevelopmentResources/DrugInteractionsLabeling/ucm110632.htm. Accessed 20 July 2015. 